\title{
A Comparison of Different Types of Burns Between Males and Females Using Clinical Results of Hospitalized Patients
}

\author{
Afsaneh Alivand, ${ }^{1,}$ Maryam Aghaamiri, ${ }^{1}$ Gelavij Mahmoodi, ${ }^{2}$ Ali Akbar Mohammadi, ${ }^{3}$ and \\ Abdolhassan Doulah ${ }^{1}$ \\ ${ }^{1}$ Department of Nursing, Faculty of Nursing and Midwifery, Ahvaz Branch, Islamic Azad University, Ahvaz, Iran \\ ${ }^{2}$ Department of Experimental Science, Kermanshah Branch, Islamic Azad University, Kermanshah, Iran \\ ${ }^{3}$ Shiraz Burn Research Center, Division of Plastic Surgery and Reconstructive Surgery, Department of Surgery, Shiraz University of Medical Sciences, Shiraz, Iran \\ "Corresponding author: Afsaneh Alivand, Department of Nursing, Faculty of Nursing and Midwifery, Ahvaz Branch, Islamic Azad University, Ahvaz, Iran. Tel: +98-9173723087, \\ Fax:+98-6133348351, E-mail: alivand2009@yahoo.comh_doulah@yahoo.com
}

Received 2016 July 03; Revised 2016 December 19; Accepted 2016 December 27.

\begin{abstract}
Background: There are significant differences between the nature of upper and lower extremity burns. This study aimed to investigate some of these differences and their possible influences on wounds healing process caused by burns on upper and lower extremities.

Methods: This study included the data from 283 patients admitted to Taleghani hospital from August 2014 to August 2015 . The patients were classified to two groups, namely patients with upper extremity burn and those with lower extremity burn. The length of hospitalization and mortality rate was also studied and compared to each other.

Results: The length of hospitalization for the female group was significantly higher than the male group $(\mathrm{P}<0.001)$. The mortality rate among female patients was much higher than that of the male patients $(30.3 \% \mathrm{vs.} 18.5 \%, \mathrm{P}=0.004)$.

Conclusions: Considering the gender effect and mortality rate, as a prognostic factor, may be useful in clinical practice.
\end{abstract}

Keywords: Burn, Clinical Results, Males, Females

\section{Background}

In spite of the lack of reliable surveys, several years of practical experiences in major burn centers have shown significant differences between the nature of upper and lower extremity burns.

Differences in prognosis, complications, and mortality rate are the most important reasons indicating the significance of conducting a study on differences between burns of upper and lower extremities (1). Drawing on the results of this study, as well as other studies, may help us develop various managerial skills that should be applied to treat burns on upper and lower extremities. Although various burn treatments are applied for burn patients, none of the instructions offered in the recent years have provided guidelines for the management of acute burns in patients (2).

According to the currently accepted practice, all burn wounds should be treated first by a surgical procedure and skin grafting to help wounds to naturally close up within three weeks (3). In fact, due to the large number of patients and lack of facilities, many health centers are yet to consider performing surgery and skin grafts as common practices to treat burn injuries. Unfortunately, seeing patients with both upper and lower extremity burns at the same time have much to do with this bitter reality. Such observations are not influenced by individual wound healing factors, which is different in each person (4).

\section{Objectives}

This study aimed to investigate some of these differences and their possible influences on wounds healing process caused by burns on upper and lower extremities. Focusing on the burn area, in the present study, we investigate results and prognosis, complications and severity, age, gender, and mortality rate of patients.

\section{Methods}

Study design and population: This retrospective survey was designed to study the length of hospitalization and mortality rate of 283 of patients with burns from August 2014 to August 2015. This study was extracted from a research project, approved by the research council of Islamic Azad University of Ahvaz.

Inclusion criteria: The inclusion criterion was admission to Taleghani hospital in Ahvaz with greater than 66\% total body surface area (TBSA) burns on upper and lower extremities. 


\subsection{Exclusion Criteria}

The exclusion criteria of this study were age of below 15 and above 45 years old, comorbidities such as diabetes mellitus, cardiovascular diseases, and hypertension, inhalation burn injury, and glasgow coma scale(GCS) score of less than 9.

\subsection{Methods}

The subjects of the study were divided to two groups. Those with greater than 66\% TBSA burn on their upper extremity (above the waistline) were assigned to the upper extremity group and the rest to the lower extremity group. Pelvic griddle (waistline) was accepted as the reference point for dividing patients into upper or lower extremity groups. Total body surface area (TBSA) was calculated according to the Berkow diagram (5).

A burn specialist doctor gathered required data from each group on a daily basis. The given groups were compared to each other in terms of variables such as mortality rate, length of hospitalization, gender, age group, and mean TBSA.

Mortality rate, length of hospitalization, and TBSA in male/female and upper/lower groups was first studied separately. Then, the upper/lower groups of male and females group were compared to each other in terms of these three variables.

\subsection{Statistical Analysis}

Statistical analysis was performed by SPSS 15.0 software using Mann-Whitney test and Chi-square statistical tests. The statistical significance level of P-value was considered less than or equal to 0.05 .

\section{Results}

Out of 283 patients included in this study, 163 were male (57.59\%). Average age of the patients was 27.4 ; there was no significant difference between male and female groups in terms of their age $(\mathrm{P}=0.0238)$. The average of TBSA was significantly greater in females than that of males $(\mathrm{P}=0.002)$. Additionally, the females' length of hospitalization was significantly greater than that of males $(\mathrm{P}<$ 0.001). The mortality rate among female patients was significantly higher than that of males $(30.3 \%$ vs. $18.5 \%, \mathrm{P}=$ $0.004)$.
4.1. Results of Comparison Between Upper and Lower Extremity Burns

The patients were divided to upper and lower extremity burn groups and were analyzed in terms of their age, TBSA, and length of hospitalization. The findings revealed that there were no significant differences between the lower and upper groups, but the length of hospitalization was significantly greater in patients with lower extremity burns $(\mathrm{P}=0.013)$. Moreover, comparing upper and lower extremity in female and male groups, separately, revealed that the length of hospitalization and TBSA were significantly different in male patients as opposed to the females.

\subsection{Results of Comparison Between Female and Male Groups}

Finally, mortality rate and TBSA were compared between males and females, separately. The male group with upper extremity burns had the lowest mortality rate compared to the other three groups (13\%). The female group with lower extremity burns had the highest mortality rate among all four groups (24.2\%) (Table 1).

\section{Discussion}

It is commonly accepted that the skin of one part of the body is different from various body areas. Furthermore, different parts of the body react to burn wounds in diverse ways. For example, the development of edema and deep vein thrombosis (DVT) is greater in the patients with lower extremity burn.

Lower extremity burns cause more complications and disabilities than upper extremity burns. Edema, which is very common in leg and foot burns, slows down the healing process of the wound and postpones the time of skin grafting (6). This may explain why, in the present study, we observed greater length of hospitalization in patients with lower extremity burns $(\mathrm{P}=0.013)$. In addition, there are multiple risk factors for patients with lower extremity burns contributing to the development of DVT and pulmonary embolism, which can be a major cause of death in these patients (7).

Lower extremity burns are susceptible to more complications and worse prognosis compared with upper extremity burns. However, according to the results of our study, the mortality rate for the male group with upper extremity burns was lower than that of the male group with lower extremity burns (13\% vs. $22.5 \%$ ). On the other hand, the mortality rate for the female group with upper extremity burns was lower than that of the female group with lower extremity burns ( $25.3 \%$ vs. $24.2 \%)$. The difference might be explained by considering that there are unique features throughout a female's skin that make 
Table 1. Difference Between Prognosis of Lower Extremity and Upper Extremity Burn Wounds

\begin{tabular}{|c|c|c|c|c|}
\hline \multirow[t]{2}{*}{ Gender } & \multirow[t]{2}{*}{ Group } & \multicolumn{2}{|c|}{ Discharge } & \multirow[t]{2}{*}{ Total } \\
\hline & & Expired & Survived & \\
\hline \multirow{4}{*}{ Male } & Upper & 16 & 107 & 123 \\
\hline & & $13.0 \%$ & $87.0 \%$ & $100.0 \%$ \\
\hline & Lower & 9 & 31 & 40 \\
\hline & & $22.5 \%$ & $77.5 \%$ & $00.0 \%$ \\
\hline \multirow{2}{*}{ Total } & 25 & 138 & 163 & \\
\hline & & $15.3 \%$ & $84.7 \%$ & $100.0 \%$ \\
\hline \multirow{4}{*}{ Female } & Upper & 22 & 65 & 87 \\
\hline & & $25.3 \%$ & $74.7 \%$ & $100.0 \%$ \\
\hline & Lower & 8 & 25 & 33 \\
\hline & & $24.2 \%$ & $75.8 \%$ & $100.0 \%$ \\
\hline \multirow{2}{*}{ Total } & 30 & 90 & 120 & \\
\hline & & $25.0 \%$ & $75.0 \%$ & $100.0 \%$ \\
\hline
\end{tabular}

their lower extremities more susceptible to complications and increase their mortality rate. Further studies should be performed to evaluate and confirm the mentioned point.

Various studies have shown that there are several differences in the skin features of each gender (8).

Additionally, a significant difference has been observed between each gender's sensitivity to skin inflammation $(9,10)$.

Previous studies have shown that gender (being male or female) influences the thickness of different layers of the skin(11). For instance, although both subcutaneous adipose tissue and epidermis are much more thicker (more than 10 folds) in females, the dermis layer is known to be thicker in males (12).

The subcutaneous adipose tissue has less vascular supply compared with other layers of the skin. Neovascularization (which originates from vessels of fascia and forms a vascular network on the surface of subcutaneous adipose tissue) is the marker of proper time for performing a skin graft. There is a direct relationship between thicker adipose tissue in females and longer duration before skin grafting. This can explain the greater length of hospitalization (and its complications such as infections and sepsis) in the female group of our study (11).

The comparison between mortality rate in male and female groups showed that the death rate in the female group is significantly higher than in the male group. Greater length of hospitalization, which was observed in the female group, may cause several complications such as malnutrition, infection, and sepsis (13). They could be con- sidered as other factors contributing to the higher mortality rate in the female group.

The skin has enzymes that transform sex hormones such as dehydroepiandrosterone into their more potent forms (dihydrotestosterone) (13).

This changes the collagen content of the skin differently in males and females (14). Indeed, collagen is the main support for the skin resistance and there is a direct and definite relationship between dermal thickness and skin collagen content (14), which means more dermal thickness and better blood supply in males. This could be another reason that explains why the healing process of wound took less time in the male group.

\subsection{Conclusion}

Waiting for a longer period before performing a skin graft in females would lead to greater length of hospitalization and increase in the complications, which may explain higher mortality rate in the female group compared with the male group.

There was no significant difference between the mortality rates of females with upper and lower extremity burns. In contrast, the mortality rates of males with lower extremity burns was higher than that of males with upper extremity burns. According to the result of this study, it is useful to consider area of burn wound injury as a prognostic factor in predicting the results of burn injuries. 


\section{Acknowledgments}

We would like to appreciate all people, who took part in this study as well those, who cooperated with us in gathering the required data. This study was the result of a research project admitted by the research council of the Islamic Azad University, Ahvaz Branch.

\section{References}

1. Mohammadi AA, Johari HG. Interesting differences between the burns involving the upper versus lower extremities. J Trauma. 2009;66(6):1746-7. doi: 10.1097/TA.0b013e3181a56f70. [PubMed: 19509642].

2. Kamolz LP, Kitzinger HB, Andel H, Frey M. The surgical treatment of acute burns. Eur J Surg. 2006;38(6):417-23. doi: 10.1007/s10353-0060296-y.

3. Munster AM. Burns of the world. J Burn Care Rehabil. 1996;17(6 Pt 1):477-84. [PubMed: 8951533].

4. Kiecolt-Glaser JK, Marucha PT, Mercado AM, Malarkey WB, Glaser R. Slowing of wound healing by psychological stress. The Lancet. 1995;346(8984):1194-6. doi: 10.1016/s0140-6736(95)92899-5.

5. Furqan $M$, Haque A. Surface area in children: a simple formula. Indian Pediatr. 2009;46(12):1085-7. [PubMed: 19430073].

6. Demling RH, Mazess RB, Witt RM, Wolberg WH. The study of burn wound edema using dichromatic absorptiometry. J Trauma. 1978;18(2):124-8. [PubMed: 633420].
7. Rue LW, Cioffi WG, Rush R, McManus WF, Pruitt BA. Thromboembolic complications in thermally injured patients. World J Surg. 1992;16(6):1151-4. doi:10.1007/bf02067085.

8. Mohammadi AA, Bakhshaeekia AR, Marzban S, Abbasi S, Ashraf AR, Mohammadi MK, et al. Early excision and skin grafting versus delayed skin grafting in deep hand burns (a randomised clinical controlled trial). Burns. 2011;37(1):36-41. doi: 10.1016/j.burns.2010.02.005. [PubMed: 20537468].

9. Thomas-Ahner JM, Wulff BC, Tober KL, Kusewitt DF, Riggenbach JA, Oberyszyn TM. Gender differences in UVB-induced skin carcinogenesis, inflammation, and DNA damage. Cancer Res. 2007;67(7):3468-74. doi: 10.1158/0008-5472.CAN-06-3798. [PubMed: 17389759].

10. Armstrong BK, Kricker A. The epidemiology of UV induced skin cancer. J Photochem Photobiol B. 2001;63(1-3):8-18. doi: 10.1016/s10111344(01)00198-1.

11. Mohammadi AA, Danesh N, Sabet B, Jalaeian H, Mohammadi MK. Selfburning: A common and tragic way of suicide in Fars province, Iran. Iran J Med Sci. 2008;33(2):110-3.

12. Azzi L, El-Alfy M, Martel C, Labrie F. Gender differences in mouse skin morphology and specific effects of sex steroids and dehydroepiandrosterone. J Invest Dermatol. 2005;124(1):22-7. doi: 10.1111/j.0022-202X.2004.23545.x. [PubMed: 15654949].

13. Mohammadi AA, Riazi H, Hasheminasab MJ, Sabet B, Mohammadi MK, Abbasi S, et al. Amniotic membrane dressing vs conventional topical antibiotic dressing in hospitalized burn patients. Iran Red Crescent Med J. 2009;11(1):66-70.

14. Brincat M, Moniz CF, Studd JW, Darby AJ, Magos A, Cooper D. Sex hormones and skin collagen content in postmenopausal women. BrMed J(Clin Res Ed). 1983;287(6402):1337-8. [PubMed: 6416400]. 